\title{
The Interaction of Protonated Octopamine and Norepinephrine with $\beta 1$ - Adrenergic Receptor: Molecular Docking and Dynamical Simulation
}

\author{
Žiko Milanović $^{1 *}$, Dušan Dimićé, Jasmina Dimitrić Marković ${ }^{2}$ Marijana Stanojević- \\ Pirković $^{3}$, Edina Avdović ${ }^{1,4}$, Zoran Marković ${ }^{4}$ \\ ${ }^{1}$ Faculty of Science, Department of Chemistry, University of Kragujevac, 12 Radoja \\ Domanovića Street, 34000 Kragujevac, Serbia \\ e-mail: ziko.milanovic@pmf.kg.ac.rs \\ 2 The Faculty of Physical Chemistry, University of Belgrade, Studentski trg 12-16, 11158 \\ Belgrade, Serbia \\ e-mail: ddimic@ffh.bg.ac.rs, markovich@ffh.bg.ac.rs \\ ${ }^{3}$ Faculty of Medical Sciences, University of Kragujevac, 69 Svetozara Markovića Street, 34000 \\ Kragujevac, Serbia \\ e-mail: marijanas14@gmail.com \\ ${ }^{4}$ Institute of Information Technologies, Department of Science, University of Kragujevac, \\ Jovana Cvijica bb, 34000 Kragujevac, Serbia \\ e-mail: zmarkovic@uni.kg.ac.rs \\ *corresponding author
}

\begin{abstract}
In the current study, the interaction mechanisms between protonated neurotransmitters: octopamine (4-(2-amino-1-hydroxyethyl)phenol) and norepinephrine (4-[(1R)-2-amino-1hydroxyethyl]benzene-1,2-diol) with the $\beta-1$ adrenergic receptor ( $\beta 1 \mathrm{AR})$ were examined by molecular docking, molecular dynamics (MD) simulations and MM/PBSA free energy calculations. The investigated receptor belongs to the G-protein coupled receptor group. The investigation was carried out at physiological $\mathrm{pH}=7.4$. It was estimated that both compounds exist in the protonated form in the water at physiological $\mathrm{pH}$. It was found that both protonated neurotransmitters established similar interactions with amino acid residues of the receptor, such as salt bridges, conventional hydrogen bonds, $\pi-\sigma$, and T-shaped $\pi-\pi$ interactions, as shown by molecular docking simulations. As the initial structures for MD simulation with a total time of $10 \mathrm{~ns}$ the most stable docking structures were used. The presented results are expected to provide some useful information for the design of specific $\beta 1 \mathrm{AR}$ agonists.
\end{abstract}

Keywords: molecular docking, molecular dynamic, MM/PBSA, neurotransmitters

\section{Introduction}

Neurotransmitters are a very important group of small organic molecules. This group of compounds regulates the transmission of electrical signals between the nerve cells, as well as between nerve cells and effector cells (Lodish et al., 2000). Norepinephrine (4-[(1R)-2-amino-1 
hydroxyethyl]benzene-1,2-diol) (Fig 1 (a)) is catecholamine neurotransmitter produced by the postganglionic sympathetic fibers (nervous system) and the core of the adrenal gland (William Tank et al.,2011). Norepinephrine takes part in many important biological processes such as motivation, memory formation, and cognition, but also accelerates heart rate and increases heart strength (Orchard, 1982). Also, noradrenaline is one of the hormones of stress (preparing the organism for fighting or escaping). Octopamine (4-(2-amino-1-hydroxyethyl)phenol) (Fig. 1(b)) is a structural analog of norepinephrine with the difference in the absence of one hydroxyl group (Foote, 1983). Octopamine is an important neurotransmitter whose function is closely related to the norepinephrine function (Farooqui, 2012). In this study, we examined the binding properties of octopamine and norepinephrine to the biologically important $\beta-1$ adrenergic receptor $(\beta 1 \mathrm{AR})$, at physiological $\mathrm{pH}$. Investigated $\beta-1$ adrenergic receptor $(\beta 1 \mathrm{AR})$ is predominantly in the cardiac tissue and cerebral cortex. This receptor stimulates the action of the salivary glands, affects the heart rate and lipolysis in the adipose tissue (Frielle et al., 1988).

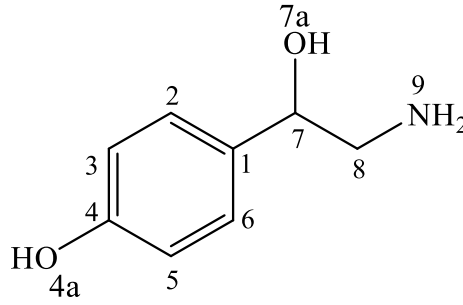

a)<smiles>NCC(O)c1ccc(O)c(O)c1</smiles>

b)

Fig.1. Structures of octopamine (a) and norepinephrine (b) with atomic numbering

A detailed description of the interactions between investigated compounds and protein can help in a better understanding of the biological effects of these components. Also, special attention is paid to the effect of an additional hydroxyl group attached to an aromatic ring in the structure of norepinephrine on the stability of the resulting protein-ligand complex.

\section{Methodology}

Molecules with more than one hydroxyl group, in aqueous solution, at physiological $\mathrm{pH}$, can exist in more than one acid-base form. Since $\mathrm{pKa}$ values can be treated as the measure of acidity, dissociation of investigated compounds depends on $\mathrm{p} K$ a values. Based on the structures of investigated compounds three $\mathrm{pKa}$ values for norepinephrine and two for octopamine can be expected (Fig 2). Thus, the portion of protonated structures (at site 9), neutral $\left(\mathrm{H}_{2} \mathrm{OCT}\right.$ and $\mathrm{H}_{2} \mathrm{NOR}$ ), mono-anionic ( $\mathrm{HOCT}^{-}$and $\mathrm{HNOR}^{-}$), and dianionic forms ( $\mathrm{NOR}^{2-}$ ) would vary depending on the $\mathrm{pH}$ of the environment. For norepinephrine, the experimentally obtained $\mathrm{pKa}$ values are $\mathrm{pKa} \mathrm{a}_{1}=8.65, \mathrm{pKa} \mathrm{a}_{2}=9.77$, and $\mathrm{pKa} \mathrm{a}_{3}=12.06$ (Álvarez-Diduk, 2015). For octopamine, experimentally $\mathrm{pKa}$ have values: $\mathrm{pKa} \mathrm{a}_{1}=8.88$ and $\mathrm{pKa} \mathrm{a}_{2}=9.53$ (Kiani, 2015). 
<smiles>NCC(O)c1ccc(Oc2ccccc2)c(O)c1</smiles><smiles>NCC(O)c1ccc(Oc2ccccc2)cc1</smiles>

Fig. 2. Different acid/base equilibria with molar fractions at physiological $\mathrm{pH}$ of octopamine (OCT) and norepinephrine (NOR)

For quantification every acido-base forms of investigated molecules are necessary to calculate the values of global formation equilibrium constants, $\beta 1, \beta 2, \beta 3$, following equations:

$$
\begin{gathered}
\beta_{1}=10^{p K a_{3}} \\
\beta_{2}=10^{p K a_{3}+p K a_{2}} \\
\beta_{3}=10^{p K a_{3}+p K a_{2}+p K a_{1}}
\end{gathered}
$$

The calculated values are necessary for the determination of the molar fractions (f). The molar fractions of different species can be estimated as follows:

$$
\begin{gathered}
f\left[A^{3-}\right]=\frac{1}{1+\beta_{1}\left[H^{+}\right]+\beta_{2}\left[H^{+}\right]^{2}+\beta_{3}\left[H^{+}\right]^{3}} \\
f\left[H^{2-}\right]=\beta_{1}\left[H^{+}\right] f\left[A^{3-}\right] \\
f\left[H_{2} A^{-}\right]=\beta_{2}\left[H^{+}\right]^{2} f\left[H A^{2-}\right] \\
f\left[H_{3} A\right]=\beta_{3}\left[H^{+}\right]^{3} f\left[H_{2} A^{-}\right]
\end{gathered}
$$

At physiological $\mathrm{pH}=7.4$, the concentration of $\mathrm{H}+$ ion is $3.98 \times 10-8 \mathrm{M}$ (Galano et al., 2015).

\subsection{Molecular docking}

Molecular docking was done by utilizing the AutoDock 4 package (Morris et al., 2009). The 3D crystal structure of $\beta 1$ AR was taken from RCSB Protein Data Bank with PDB code 4LNW (Souza et al.,2014). The Discovery Studio 4.0 (BIOVIA Discovery Studio 2016) was used to prepare the PDB file (to select the needed chains, delete multiple ligands, and non-protein parts). The investigated molecules (Fig.3) were optimized in the Gaussian 09 program package at B3LYP-D3BJ/6-311++G(d,p) level theory (Frisch et al., 2010). Before the docking runs, Autodock tools (ADT) was used to add Kollman united atom charges and polar hydrogen atoms to the prepared receptor. The grid box of dimensions $60 \times 60 \times 60 \AA$ along the XYZ directions with a grid spacing of $0.375 \AA$ was created using the AutoGrid module. The Lamarckian 
Genetic Algorithm (LGA) was selected for the search for the conformers with the lowest energy. During the docking process, ten independent docking runs were completed for the ligand. The maximum numbers of generation and evaluation were set at 27000 and 2500000 , respectively.

\subsection{Molecular dynamic}

The docked complexes with the lowest binding energy and inhibition constant were subjected to MD simulations using GROMACS 5.1.5. a software package (Abraham et al., 2015). The topologies of the octopamine, norepinephrine, and $\beta 1 \mathrm{AR}$ receptor were generated by the means of the CHARMM36 force field (Vanommeslaeghe et al., 2010). The CGenFF server (Yu, 2012) was used for the generation of topologies and coordinate files of neurotransmitters. The partial charges were calculated using the Gaussian09 program package and B3LYP-D3BJ theoretical method in conjunction with 6-311++G (d,p) basis set (Frisch et al., 2009). After the successful generation of topology for receptor and neurotransmitters, all docked complexes were immersed in the TIP3 solvation model (Zielkiewicz, 2005) and the systems were neutralized by chloride ions $(0.15 \mathrm{M} \mathrm{KCl})$ using Monte-Carlo Ion Placing Method (Hünenberger, 1999). The neutralized systems were energetically minimized by steepest descent and conjugate gradient algorithms with up to a tolerance of $1000 \mathrm{~kJ} \mathrm{~mol}^{-1} \mathrm{~nm}^{-1}$ during of 50000 steps. The equilibration process was carried out separately in NVT (constant volume) as well as NPT (constant pressure) ensemble conditions, each with a 1 ns time scale. In both conditions, the temperature of the system was maintained at $298 \mathrm{~K}$ using the Berendsen weak coupling method. In addition, the pressure was maintained at 1 bar by utilizing Parrinello-Rahman barostat in constant pressure ensemble (Berendsen et al., 1984). Finally, the MD production process was carried out in the NPT ensemble using the LINCS algorithm for $10 \mathrm{~ns}$ time scale including a modified Berendsen thermostat $\left(\tau_{\mathrm{T}}=1 \mathrm{ps}\right)$ and a Parrinello-Rahman barostat $\left(\tau_{\mathrm{P}}=2 \mathrm{ps}\right)$ (Hess, 1997). The RMSD (Root Mean Square Deviations), Radius of Gyration $\left(\mathrm{R}_{\mathrm{g}}\right)$, and H-bonds between the proteinligand complexes were analyzed.

\subsection{Calculation of Binding Free Energies by MM/PBSA}

Molecular mechanics Poisson-Boltzmann surface area (MM/PBSA) method is an attractive approach to estimate the free energy of binding and has been increasingly used in the study of interactions between biologically important macromolecules and small molecules. The binding between protein or another biomolecule $(\mathrm{R})$ and ligand $(\mathrm{L})$ can be described as:

$$
L+R \rightarrow R L
$$

The binding free energy, $\Delta G_{\text {bind }}$, in a solvent, is estimated by the following equation:

$$
\Delta G_{\text {bind }}=G_{\text {complex }}-\left(G_{\text {receptor }}+G_{\text {ligand }}\right)
$$

where $G_{\text {complex }}$ is the total free energy of protein-ligand complex and $G_{\text {receptor }}$ and $G_{\text {ligand }}$ are total free energies of receptor and ligand in a solvent, respectively (Kollman et al., 2000). The binding free energy $\left(\Delta G_{\text {bind }}\right)$ is a set of different energy terms. The calculated binding free energy $\left(\Delta G_{\text {bind }}\right)$ is the sum of average molecular mechanics potential energy $\left(\Delta E_{\mathrm{MM}}\right)$ interaction and solvation free energy $\left(\Delta G_{\text {sol }}\right)$, reduced by the entropic contribution $(T \Delta S$ ) (Gohlke et al., 2003):

$$
\Delta G_{\mathrm{X}}=\Delta H-T \Delta S \approx \Delta E_{\mathrm{MM}}+\Delta G_{\mathrm{sol}}-T \Delta S
$$

In equation $10, \mathrm{x}$ is the receptor-ligand complex or individual receptor and ligand. $\Delta \mathrm{Gx}$ is the average vacuum potential energy, $\triangle \mathrm{EMM}$ is calculated based on the molecular mechanics 
(MM) force-field parameters, and it presents the sum of energy of bonded interactions ( $\Delta$ Ebonded) as well as nonbonded interactions ( $\Delta$ Enonbonded), and it can be written as:

$$
\Delta E_{\mathrm{MM}}=\Delta E_{\mathrm{bonded}}+\Delta E_{\mathrm{nonbonded}}=\Delta E_{\mathrm{bonded}}+\Delta E_{\mathrm{ELE}}+\Delta E_{\mathrm{VDW}}
$$

The energy of bonded interactions ( $\left.\Delta E_{\text {bonded }}\right)$ is a parameter that consists of the bond, angle, dihedral, and improper interactions. The nonbonded interactions energy ( $\left.E_{\text {nonbonded }}\right)$ is the sum of electrostatic $\left(E_{\text {elec }}\right)$ and van der Waals $\left(E_{\mathrm{VDW}}\right)$ interactions. In this study, these parameters were created by the Coulomb and Lennard-Jones $(\mathrm{LJ})$ potential functions, respectively. The energy of bonded interactions ( $\left.\Delta E_{\text {bonded }}\right)$ is always taken as zero (Hornak, 2006). The solvation free energy $\left(\Delta G_{\text {sol }}\right)$ is the sum of electrostatic polar $\left(\Delta G_{\text {polar }}\right)$ and nonelectrostatic non-polar $\left(\Delta G_{\text {nonpolar }}\right)$ energy terms:

$$
\Delta G_{\mathrm{sol}}=\Delta G_{\mathrm{polar}}+\Delta G_{\text {nonpolar }}
$$

The electrostatic polar term, $\mathrm{G}_{\text {polar }}$, is estimated by solving the Poisson-Boltzmann (PB) equation:

$$
\nabla \cdot[\varepsilon(\mathrm{r}) \mid \nabla \cdot \varphi(\mathrm{r})]-\varepsilon(\mathrm{r}) \mathrm{k}(\mathrm{r})^{2} \sinh [\varphi(\mathrm{r})]+\frac{4 \pi \rho^{\mathrm{f}}(\mathrm{r})}{\mathrm{kT}}=0
$$

In equation 13, $\varphi(r)$ is electrostatic potential, $\varepsilon(r)$ is the dielectric constant, and $\rho^{\mathrm{f}}(\mathrm{r})$ is the fixed charge density. The term $\mathrm{k}(\mathrm{r})^{2}$ is related to the reciprocal of Debye length which is dependent on the ionic strength of the solution (Srinivasan, 1998).

This model is based on the assumption that the Solvent-accessible surface area (SASA) models is linearly dependent on the $\mathrm{G}_{\text {nonpolar }}$ term and can thus be calculated as follows:

$$
G_{\text {nonpolar }}=\gamma A+b
$$

where $\gamma$ is a coefficient related to the surface tension of the solvent, $A$ is SASA, and $b$ is a fitting parameter (Wang, 2000). All described thermodynamic parameters were calculated using the g_mmpbsa package (Kumari, 2014).

\section{Results and discussion}

At physiological $\mathrm{pH}$, in aqueous solution, the dominant species for octopamine and norepinephrine are the protonated ones (Fig. 2), with a population of $\sim 96.77 \%$ and $\sim 94.65 \%$, respectively. For this reason, only the protonated forms of these compounds were examined. The protonated compounds (Fig. 3) contain several polar groups, namely the hydroxyl group ($\mathrm{OH})$ as well as the protonated amino group $\left(-\mathrm{NH}_{3}{ }^{+}\right)$. The atoms of mentioned functional groups represent donor or acceptor atoms in interactions with different amino acids of the $\beta 1 \mathrm{AR}$ receptor. 


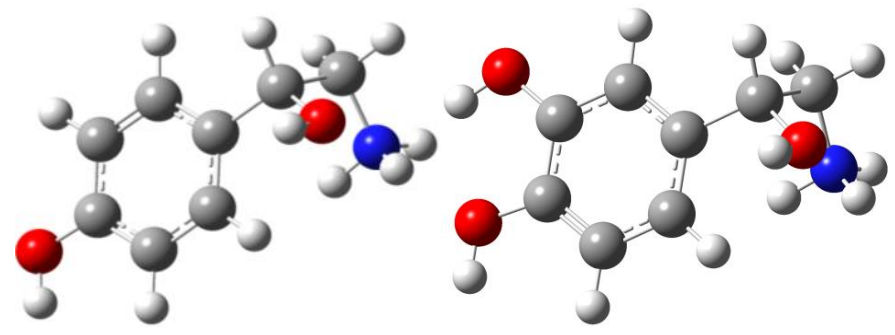

Fig. 3.The optimized protonated structure of octopamine (left) and norepinephrine (right) obtained at the B3LYP-D3BJ/6-311++G(d,p) level of theory

Table 1 shows the results for binding energy $\left(\Delta G_{\text {bind }}\right)$ and inhibition constants $\left(K_{\mathrm{i}}\right)$ of the most stable structure obtained by molecular docking. Also, the same table shows interactions and distances between amino acids in the receptor active pocket and neurotransmitters. Octopamine and norepinephrine interact with $\beta 1 \mathrm{AR}$ receptor with binding energies of $-19.96 \mathrm{~kJ}$ $\mathrm{mol}^{-1}$ and $-22.80 \mathrm{~kJ} \mathrm{~mol}^{-1}$ and inhibition constants of 316.15 and $100.56 \mu \mathrm{M}$. Negative values of binding energy and low values of inhibition constant are considered indicative of a stable binding. A more favorable binding of protonated norepinephrine to the receptor is due to the presence of one more hydroxyl group in meta position which enhances the biological activity of this compound.

\begin{tabular}{|c|c|c|c|c|c|}
\hline Complex: & Interactions: & Types of interactions: & $\AA$ & $\begin{array}{c}\Delta G_{\text {bind }} \\
\left(\mathrm{kJ} \mathrm{mol}^{-1}\right)\end{array}$ & $\begin{array}{c}K_{\mathrm{i}} \\
(\mu \mathrm{M})\end{array}$ \\
\hline \multirow{10}{*}{$\begin{array}{l}\text { B1AR- } \\
\text { OCT }\end{array}$} & OCT:H -A:ASP121:O & Salt Bridge;Attractive Charge & 1.84 & \multirow{10}{*}{-19.96} & \multirow{10}{*}{316.15} \\
\hline & A:ASN335:H - OCT:O & Conventional Hydrogen Bond & 2.16 & & \\
\hline & OCT:H - A:ASP121:O & Conventional Hydrogen Bond & 2.19 & & \\
\hline & OCT:H - A:ASP121:O & Conventional Hydrogen Bond & 2.18 & & \\
\hline & OCT:H - A:ASP121:O & Conventional Hydrogen Bond & 2.23 & & \\
\hline & OCT:H - A:ASP121:O & $\begin{array}{c}\text { Conventional Hydrogen } \\
\text { Bond }\end{array}$ & 2.89 & & \\
\hline & OCT:H - A:ASP87:O & Conventional Hydrogen Bond & 1.81 & & \\
\hline & A:VAL90:CG2 - OCT & $\pi-\sigma$ & 3.46 & & \\
\hline & A:TRP303 - OCT & $\pi$ - $\pi$-T-shaped & 5.15 & & \\
\hline & A:TRP303 - OCT & $\pi$ - $\pi$-T-shaped & 5.07 & & \\
\hline \multirow{12}{*}{$\begin{array}{l}\text { B1AR- } \\
\text { NOR }\end{array}$} & NOR:H - A:ASP121:O & Salt Bridge;Attractive Charge & 2.01 & \multirow{12}{*}{-22.80} & \multirow{12}{*}{100.56} \\
\hline & A:SER128:H- NOR:O & Conventional Hydrogen Bond & 1.63 & & \\
\hline & A:ASN335:H - NOR:O & Conventional Hydrogen Bond & 1.84 & & \\
\hline & NOR:H - A:ASP121:O & Conventional Hydrogen Bond & 2.16 & & \\
\hline & NOR:H - A:ASP121:O & Conventional Hydrogen Bond & 1.77 & & \\
\hline & NOR:H - A:ASP87:O & Conventional Hydrogen Bond & 2.28 & & \\
\hline & NOR:H - A:ASP87:O & Conventional Hydrogen Bond & 2.05 & & \\
\hline & NOR:H - A:SER336:O & Conventional Hydrogen Bond & 2.36 & & \\
\hline & A:VAL90:CG2 - NOR & $\pi-\sigma$ & 3.35 & & \\
\hline & A:TRP303:CZ2 - NOR & $\pi-\sigma$ & 3.47 & & \\
\hline & A:TRP303-NOR & $\pi$ - $\pi$-T-shaped & 5.02 & & \\
\hline & A:TRP303 - NOR & $\pi$ - $\pi$-T-shaped & 4.87 & & \\
\hline
\end{tabular}

Table 1. Types, the distance of interactions and thermodynamic parameters generated after molecular docking simulation 
The most favorable docking positions are presented in Fig. 4. Amino acid ASP 121 builds conventional hydrogen bonds between the oxygen atom of the carbonyl group and the hydrogen atom of $-\mathrm{NH}_{2}$ and $-\mathrm{OH}$ groups of octopamine $(2.18,2.19,2.23$ and $2.89 \AA)$ and norepinephrine (1.77 and $2.16 \AA$ ). Amino acid ASN 335 establishes conventional hydrogen bonds through the hydrogen atom of $-\mathrm{NH}_{2}$ group and the oxygen atom of the hydroxyl group of octopamine

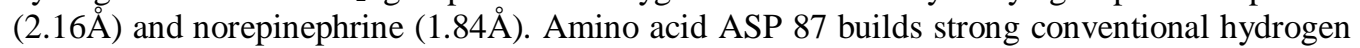
bonds between the oxygen atom of the carbonyl group and the hydrogen atom of -OH group of octopamine $(1.81 \AA)$ and norepinephrine $(2.05$ and $2.28 \AA)$. It should be noted that bonds are formed with both hydroxyl groups of norepinephrine which additionally stabilizes the complex structure. The hydrogen bond is also established between the hydroxyl group of SER 336 and the oxygen atom of norepinephrine $(1.63 \AA)$. Hydrophobic interactions involving aromatic rings are ubiquitous in chemical and biological systems. Amino acid VAL 90 builds $\pi-\sigma$ interaction

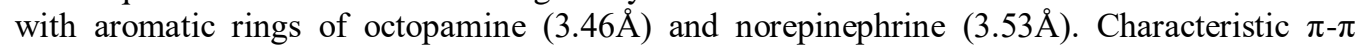
interactions reduce the aromaticity of the ring and improve the physicochemical properties of ligand, such as solubility. Amino acid TRP 303 also forms two T-shaped $\pi-\pi$ interactions, through indole ring, with the aromatic ring of octopamine (5.07 and $5.15 \AA$ ) and norepinephrine (4.87 and $5.02 \AA$ ).
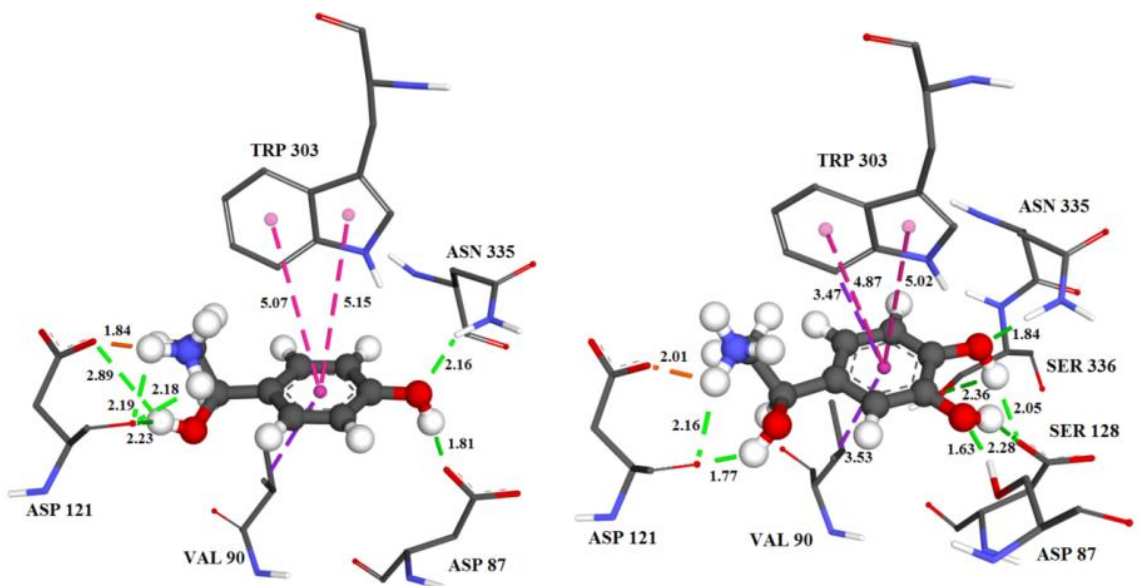

$\square$ Salt Bridge $\square$ Conventional Hydrogen Bond $\square$ Pi-Sigma $\square$ Pi-Pi T-shaped

Fig. 4. The best docking position of octopamine (left) and norepinephrine (right) to the $\beta-1$ adrenergic receptor $(\beta 1 \mathrm{AR})$ with showing the interacting amino acid residues

An interesting and significant interaction in analyzed systems is the salt bridge. The salt bridge is a combination of two non-covalent interactions - two ionized molecules mixing two contributions: hydrogen bonding and ionic bonding (Kurczab, 2018), and it is presented in Figure 5. Ion pairing is one of the most important non-covalent interactions in biological macromolecules. The studies showed that salt bridge interactions increase the thermal stability of proteins. This interaction can be observed between the partially negative carboxylate oxygen atom of amino acid ASP 121 and proton of $\mathrm{NH}_{3}{ }^{+}$group of octopamine $(1.84 \AA)$ and norepinephrine $(2.01 \AA)$. The relatively short distance indicates that the proton may migrate from an amino group of ligands to side chain of amino acid ASP 121 (Fig. 5). 

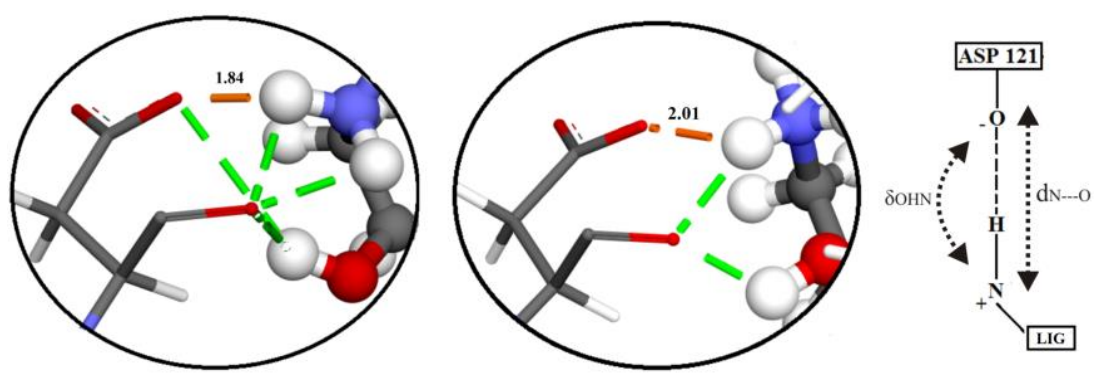

Fig. 5. Illustration of the salt bridge occurring in $\beta 1 \mathrm{AR}-\mathrm{OCT}$ (left) and $\beta 1 \mathrm{AR}-\mathrm{NOR}$ (right) complexes with interatomic distance

The structures of receptor and receptor-ligand complexes ( $\beta 1 \mathrm{AR}-\mathrm{OCT}$ and $\beta 1 \mathrm{AR}-\mathrm{NOR})$ with the lowest binding energy from molecular docking results were used for MD simulation.

Analysis of the results presented in Figure 6 obtained by MD simulation indicates a partial structural similarity with the initial conformations obtained by docking. As for the most stable docking structures, the amino acid ASP121 forms a hydrogen bond through the carbonyl group with the hydrogen atom of the hydroxyl group of octopamine $(1.61 \AA)$ and norepinephrine $(1.72$ $\AA)$. Also, the oxygen atom of the $\mathrm{C}=\mathrm{O}$ group of amino acid ASP 87 builds conventional hydrogen bonds with the hydroxyl group of octopamine $(2.72 \AA)$ and norepinephrine $(2.08$ and $2.55 \AA$ ). The oxygen atom of residue ASP 121 is involved in creating salt bridge interaction with the hydrogen atom of the amino group of octopamine (1.91 $⿱$ ) $)$ and norepinephrine $(1.77$ $\AA)$. Hydrophobic $\pi-\sigma$ interactions between VAL 90 and aromatic rings of octopamine (4.61 $\AA$ ) and norepinephrine $(4.72 \AA)$ are also present. The aromatic ring of amino acid TRP 303 interacts with the aromatic ring of octopamine through $\mathrm{T}$-shaped $\pi-\pi$ interactions. This interaction in the $\beta 1$ AR-NOR complex is replaced by amide- $\pi$ interaction with CYS $124(4.80$ $\AA$ ). Based on these results it can be concluded that the obtained results of molecular docking and molecular dynamics simulations of $\beta 1 \mathrm{AR}-\mathrm{OCT}$ and $\beta 1 \mathrm{AR}-\mathrm{NOR}$ confirm the existence of relatively same binding modes. This difference can be explained by the effect of solvent, counterions, and low energy of interactions. Also, the difference is due to the fact that the MD simulation was performed at a constant temperature $(298.15 \mathrm{~K})$ and pressure (1 bar).
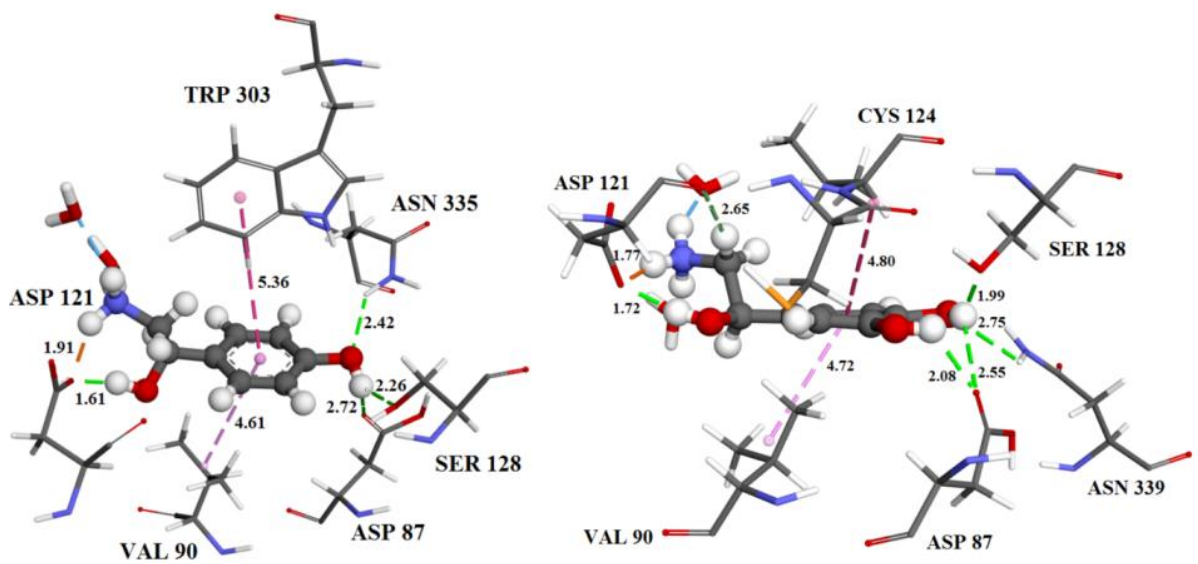

Salt Bridge $\quad$ Conventional Hydrogen Bond

Pi-Sigma

Pi-Pi T-shaped

Amide-Pi Stacked

Fig. 6. The $\beta 1$ AR-OCT (left) and $\beta 1$ AR-NOR(right) complexes obtained after $10 \mathrm{~ns}$ molecular dynamics simulation 
In order to examine conformational variations of $\beta 1 \mathrm{AR}$ receptor and corresponding $\beta 1 \mathrm{AR}-$ OCT and $\beta 1 A R-N O R$ complexes the following parameters were employed: Root Mean Square Deviation (RMSD), Root Mean Square Fluctuation (RMSF) and Radius of gyration (Rg). Figure 7 shows the RMSD values of the $\beta 1 \mathrm{AR}$ receptor backbone $(\mathrm{C}-\mathrm{C} \alpha-\mathrm{N})$ with and without octopamine and norepinephrine, as a function of the simulations times (0 to $10000 \mathrm{ps}$ ). During the initial simulation time, the RMSD values of all structures showed an up drift. The receptor $\beta 1 \mathrm{AR}$ and complexes with octopamine and norepinephrine have average RMSD fluctuation values of $0.17 \pm 0.06,0.23 \pm 0.04$ and $0.19 \pm 0.02 \mathrm{~nm}$, respectively. The corresponding $\beta 1 \mathrm{AR}-$ OCT complex has an average RMSD value higher than the $\beta 1 \mathrm{AR}$ receptor, but the $\beta 1 \mathrm{AR}$ receptor shows greater oscillation than the corresponding complex during simulation time. Also, the $\beta 1$ AR-NOR complex has a slightly higher average RMSD value and fewer oscillations than the free receptor. Based on obtained results it can be concluded that after the formation of complexes, proteins in $\beta 1 \mathrm{AR}-\mathrm{OCT}$ and $\beta 1 \mathrm{AR}-\mathrm{NOR}$ have a slight decrease in rigidity. This is a consequence of structural changes after the binding of octopamine and norepinephrine and the effect of the solvent.
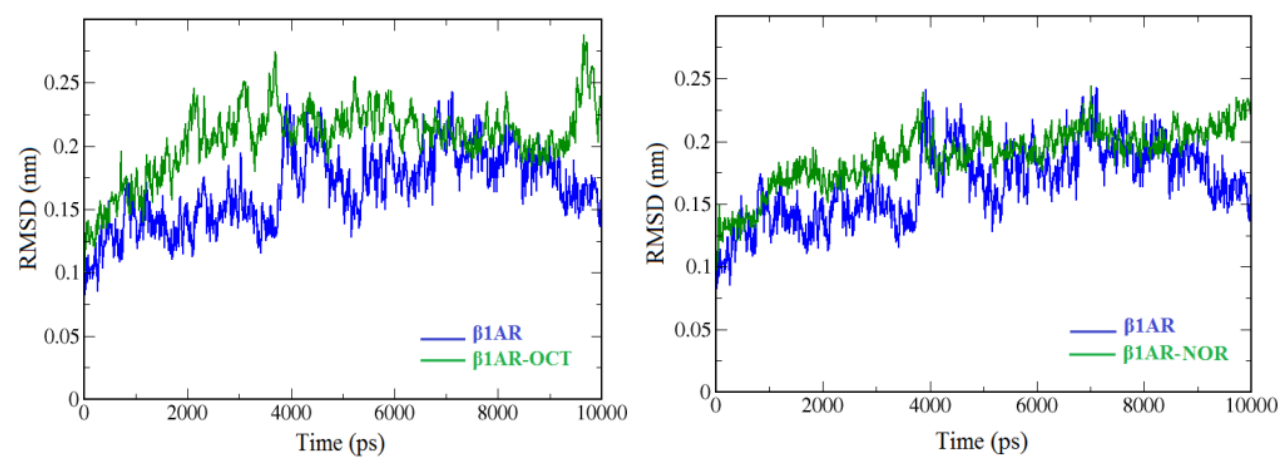

Fig. 7. RMSD plots of $\beta 1 \mathrm{AR}$ receptor backbone $(\mathrm{C}-\mathrm{C} \alpha-\mathrm{N})$ with and without neurotransmitters during $10 \mathrm{~ns}$ MD simulation

Figure 8 gives the RMSF plots for the $\beta 1 \mathrm{AR}$ receptor and corresponding complexes. These plots explain the effect of individual amino acid residues on the local protein flexibility. When the presented plots are analyzed, it is evident that in the all complexes amino acid residues in binding mode fluctuate with lower intensity with regard to the free receptor. Amino acids ASP 87, VAL 90, ASP 121, SER 128, in active site of $\beta 1$ AR-OCT $(0.047,0.051,0.053$ and 0.054 $\mathrm{nm}$, respectively) and active site of $\beta 1$ AR-NOR $(0.057,0.062,0.060$ and $0.055 \mathrm{~nm}$, respectively) complexes show lower intense oscillations than in the free protein $\beta 1$ AR $(0.099$, $0.069,0.078$ and $0.058 \mathrm{~nm}$, respectively). Low RMSF values indicate that amino acids in the active site of the receptor are relatively rigid for all investigated systems. 

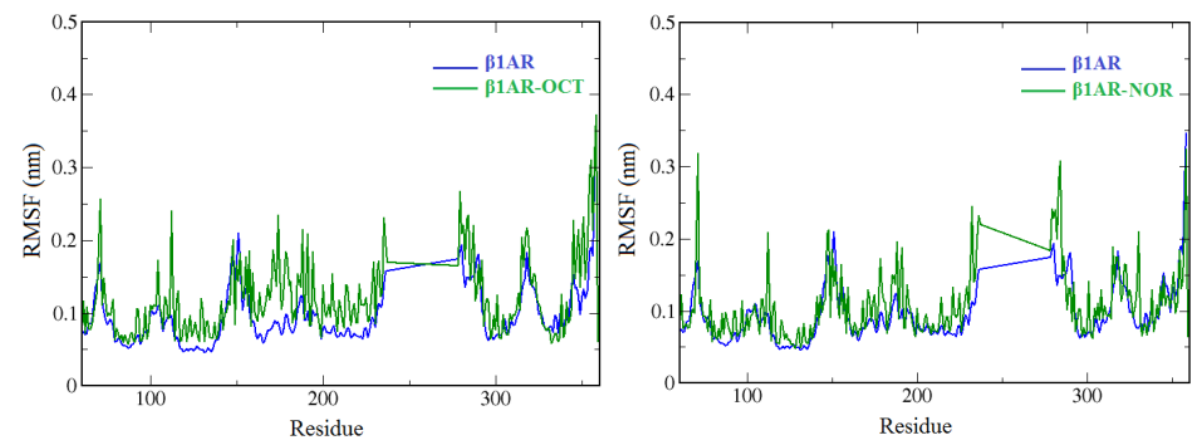

Fig. 8. RMSF plots residual fluctuations of $\beta 1 A R$ receptor with and without neurotransmitters during $10 \mathrm{~ns}$ MD simulation

The $\mathrm{Rg}$ values of the $\beta 1 \mathrm{AR}$ receptor and $\beta 1 \mathrm{AR}-\mathrm{OCT}$ and $\beta 1 \mathrm{AR}-\mathrm{NOR}$ complexes were calculated and presented in Figure 9. During simulation time (10 ns) the average Rg value of $\beta 1$ AR-OCT $(2.12 \pm 0.01 \mathrm{~nm})$ and $\beta 1$ AR-NOR $(2.10 \pm 0.01 \mathrm{~nm})$ complexes was similar to that of $\beta 1 \mathrm{AR}$ protein $(2.11 \pm 0.01 \mathrm{~nm})$. Similar oscillations and fluctuation $\mathrm{Rg}$ values, during simulation time, indicate that octopamine and norepinephrine do not disturb significantly the structure of $\beta 1 \mathrm{AR}$ protein.
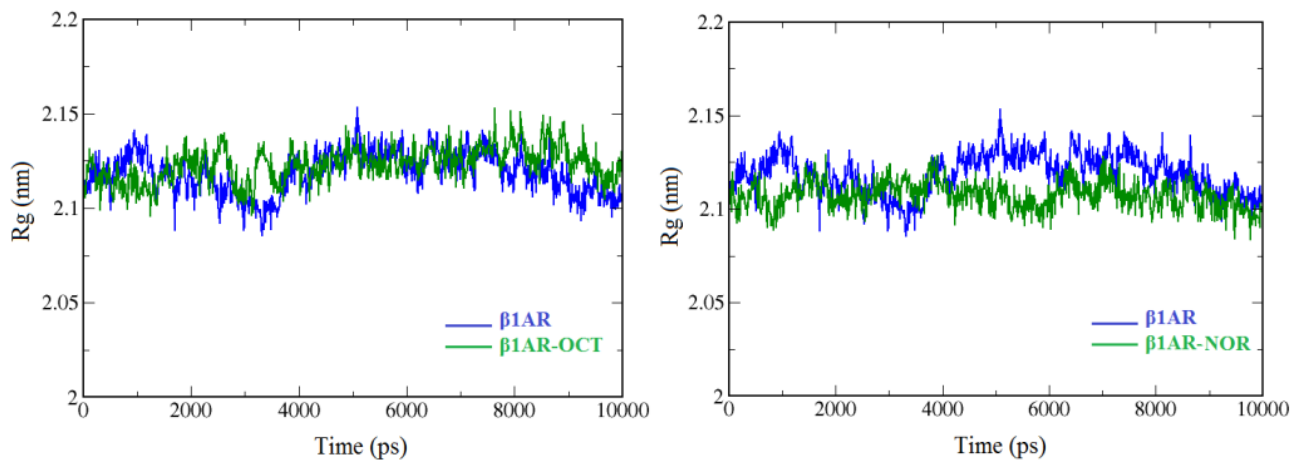

Fig. 9. Rg plots of $\beta 1 \mathrm{AR}$ receptor with and without neurotransmitters during $10 \mathrm{~ns}$ MD simulation

The affinity of the neurotransmitters to bind to the $\beta 1 \mathrm{AR}$ receptor was estimated by MM/PBSA protocol. The thermodynamic parameters are presented in table 2. Figure 10 shows the change of important thermodynamic parameters during $10 \mathrm{~ns}$ simulation time. The energy contributions of Van der Waals interactions $\left(E_{\mathrm{VDW}}\right)$ to total binding free energy, $\Delta G_{\text {bind }}$, for $\beta 1 \mathrm{AR}-\mathrm{OCT}$, and $\beta 1 \mathrm{AR}-\mathrm{NOR}$ complexes are -74.4 and $-85.1 \mathrm{~kJ} \mathrm{~mol}^{-1}$, respectively. This is in line with the fact that hydrophobic interactions are the weakest of all observed interactions. The contribution of the energy of electrostatic interactions $\left(\mathrm{E}_{\text {elec }}\right)$ to the total binding free energy for $\beta 1$ AR-OCT $\left(-237.2 \mathrm{~kJ} \mathrm{~mol}^{-1}\right)$ and $\beta 1$ AR-NOR $\left(-230.3 \mathrm{~kJ} \mathrm{~mol}^{-1}\right)$ is very significant. The high values are a consequence of the number of conventional hydrogen bonds formed between the polar groups of the neurotransmitters and the amino acid residues of the $\beta 1 \mathrm{AR}$ receptor. Also, the establishment of salt bridge interactions between the hydrogen of the protonated amino group and the oxygen of the carbonyl group of amino acids affect increases in the electrostatic term in total binding free energy. The values of nonpolar free energy for $\beta 1$ AR-OCT $(-11.7 \mathrm{~kJ}$ $\left.\mathrm{mol}^{-1}\right)$ and $\beta 1$ AR-NOR $\left(-11.8 \mathrm{~kJ} \mathrm{~mol}^{-1}\right)$ complexes, which were calculated from the SASA 
algorithm, slightly contribute to total binding free energy. The electrostatic component of the solvation free energy has high positive values which indicate that these values impair the binding process. Based on the obtained results, it can be concluded that the vacuum potential energy (van der Waals and electrostatic interactions) are major contributors to the total binding free energy. By comparing $\Delta G_{\text {bind }}$ value, it can be concluded that norepinephrine $\left(-49.7 \mathrm{~kJ} \mathrm{~mol}^{-}\right.$ $\left.{ }^{1}\right)$ forms a more stable complex than octopamine $\left(-47.3 \mathrm{~kJ} \mathrm{~mol}^{-1}\right)$ with $\beta 1 \mathrm{AR}$ receptor. This is due to the presence of additional polar hydroxyl group.

\begin{tabular}{cccccc}
\hline Complex & $\begin{array}{c}\Delta E_{\text {elec }} \\
\left(\mathrm{kJ} \mathrm{mol}^{-1}\right)\end{array}$ & $\begin{array}{c}\Delta E_{\mathrm{VDW}} \\
\left(\mathrm{kJ} \mathrm{mol}^{-1}\right)\end{array}$ & $\begin{array}{c}\Delta G_{\mathrm{polar}} \\
\left(\mathrm{kJ} \mathrm{mol}^{-1}\right)\end{array}$ & $\begin{array}{c}\Delta G_{\text {nonpolar }} \\
\left(\mathrm{kJ} \mathrm{mol}^{-1}\right)\end{array}$ & $\begin{array}{c}\Delta G_{\text {binding }} \\
\left(\mathrm{kJ} \mathrm{mol}^{-1}\right)\end{array}$ \\
\hline $\boldsymbol{\beta 1 A R - O C T}$ & $-237.2 \pm 23.4$ & $-74.4 \pm 12.1$ & $370.6 \pm 24.4$ & $-11.7 \pm 0.6$ & $-47.3 \pm 20.8$ \\
\hline $\boldsymbol{\beta 1 A R - N O R}$ & $-230.3 \pm 21.4$ & $-85.1 \pm 13.2$ & $373.8 \pm 21.6$ & $-11.8 \pm 0.6$ & $-49.7 \pm 20.1$ \\
\hline
\end{tabular}

Table 2. Important thermodynamic parameters during $10 \mathrm{~ns}$ MD simulation generated with MM/PBSA protocol
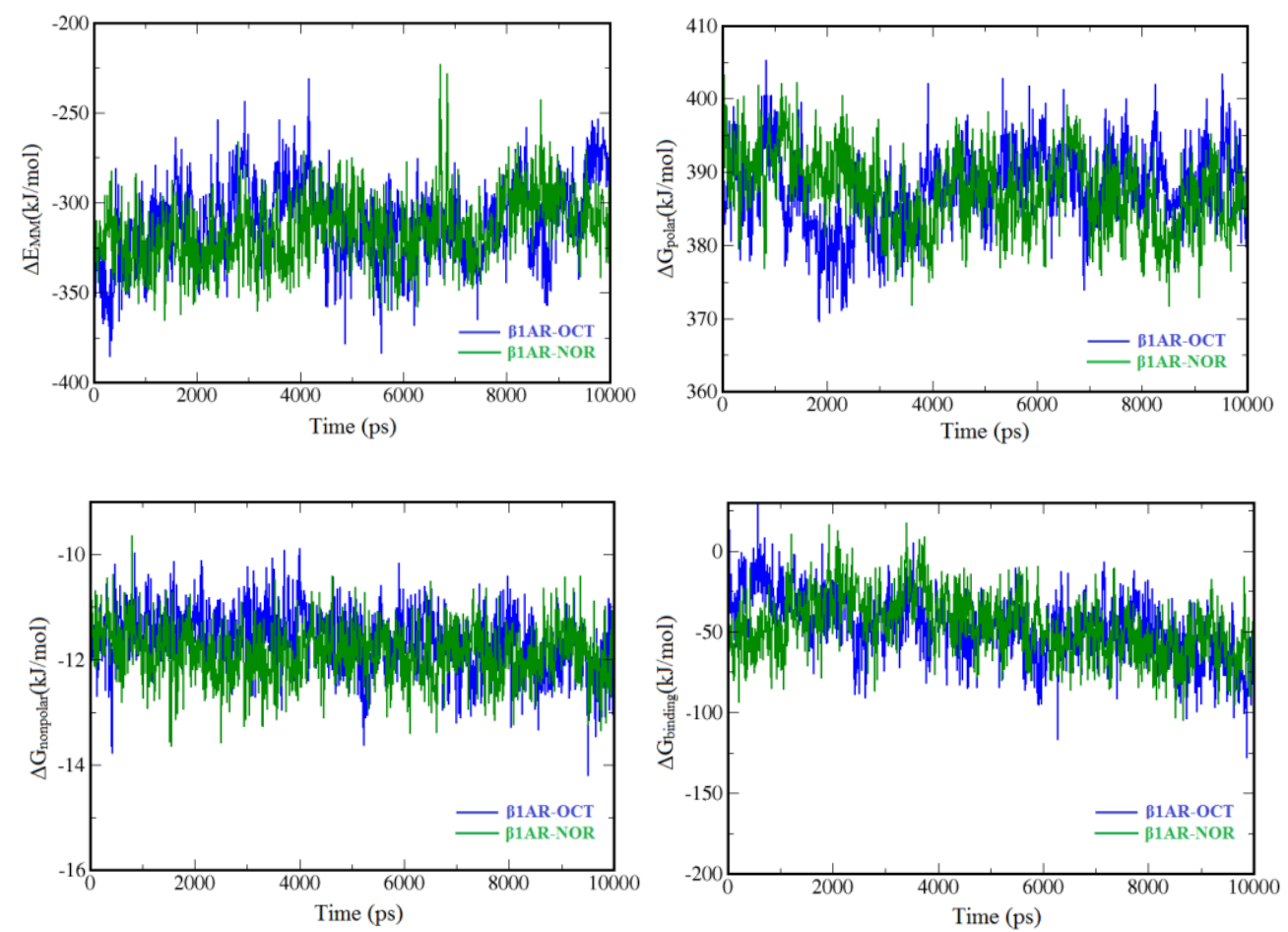

Fig. 10. Important thermodynamic parameters during $10 \mathrm{~ns}$ MD simulation

\section{Conclusions}

Within this study, the interactions of octopamine and norepinephrine with $\beta-1$ adrenergic receptor $(\beta 1 \mathrm{AR})$ receptor under physiological conditions (aqueous solution and $\mathrm{pH}=7.4$ ) were 
examined. Based on the $\mathrm{pKa}$ values, it was estimated that more than $96.77 \%$ of octopamine and $94.65 \%$ of norepinephrine are in the protonated form. The results of molecular docking simulation indicate that a more stable complex is formed with norepinephrine, with a binding energy of $-22.80 \mathrm{~kJ} \mathrm{~mol}^{-1}$ and inhibition constant of $100.56 \mu \mathrm{M}$ which is a consequence of the presence of additional hydroxyl group. The obtained interactions were more numerous for the $\beta 1$ AR-NOR complex, although the type and strength of interactions remained the same. The same type of interactions, namely hydrogen bonds, $\pi-\pi, \pi-\sigma$, and salt bridges, were observed for molecular dynamics simulations. The rigidity of formed complexes was proven when the flexibility of amino acid residues was compared to free protein as quantified by Root Mean Square Deviation (RMSD) and Root Mean Square Fluctuation (RMSF). The most important contribution to the total binding energy in both complexes came from electrostatic interactions and hydrogen bonds, followed by van der Waals and nonbonding interactions. These results prove that the hydroxyl group in meta-position could possibly have important biological implications, especially when binding to proteins is concerned.

Acknowledgments: This paper was supported by the Serbian Ministry of Education, Science and Technological Development (Agreements No. 451-03-68/2020-14/200122; 451-0368/2020-14/200378).

\section{References}

Abraham M. J, Murtola T, Schulz R, Páll S, Smith J. C, Hess B, Lindahl, E. (2015). GROMACS: High-performance molecular simulations through multi-level parallelism from laptops to supercomputers. SoftwareX, 1, 19-25.

Álvarez-Diduk R, Galano A. (2015). Adrenaline and noradrenaline: protectors against oxidative stress or molecular targets? The Journal of Physical Chemistry B, 119(8), 3479-3491.

Berendsen H. J., Postma J. V, van Gunsteren W. F, DiNola A. R. H. J, Haak J. R. (1984). Molecular dynamics with coupling to an external bath. The Journal of chemical physics, 81(8), 3684-3690.

Farooqui T. (2012). Review of octopamine in insect nervous systems. Open access insect physiol, 4, 1-17.

Foote S. L, Bloom F. E, Aston-Jones G. (1983). Nucleus locus ceruleus: new evidence of anatomical and physiological specificity. Physiological reviews, 63(3), 844-914.

Frielle T, Kobilka B, Lefkowitz R. J, Caron M. G. (1988). Human $\beta 1$-and $\beta 2$-adrenergic receptors: structurally and functionally related receptors derived from distinct genes. Trends in neurosciences, 11(7), 321-324.

Frisch M. J, Trucks G. W, Schlegel H. B, Scuseria G. E, Robb M. A, Cheeseman J. R, Millam J. M. (2003). Revision B. Gaussian. Inc., Pittsburgh PA.

Galano A, Alvarez-Idaboy J. R. (2013). A computational methodology for accurate predictions of rate constants in solution: Application to the assessment of primary antioxidant activity. Journal of computational chemistry, 34(28), 2430-2445.

Gohlke H, Kiel C, Case D. A. (2003). Insights into protein-protein binding by binding free energy calculation and free energy decomposition for the Ras-Raf and Ras-RalGDS complexes. Journal of molecular biology, 330(4), 891-913.

Hess B, Bekker H, Berendsen H. J, Fraaije J. G. (1997). LINCS: a linear constraint solver for molecular simulations. Journal of computational chemistry, 18(12), 1463-1472.

Hornak V, Abel R, Okur A, Strockbine B, Roitberg A, Simmerling C. (2006). Comparison of multiple Amber force fields and development of improved protein backbone parameters. Proteins: Structure, Function, and Bioinformatics, 65(3), 712-725. 
Hünenberger P. H, McCammon J. A. (1999). Ewald artifacts in computer simulations of ionic solvation and ion-ion interaction: a continuum electrostatics study. The Journal of chemical physics, 110(4), 1856-1872.

Kiani F, Abbaszadeh M, Pousti, M, Koohyar, F. (2015). Ab initio and DFT studies on ionization of octopamine and 6-aminopenicillanic acid in aqueous solution.

Kollman P. A, Massova I, Reyes C, Kuhn B, Huo S, Chong L, Donini O. (2000). Calculating structures and free energies of complex molecules: combining molecular mechanics and continuum models. Accounts of chemical research, 33(12), 889-897.

Kumari R, Kumar R.(2014). Open Source Drug Discovery Consortium, \& Lynn, A. g_mmpbsa A GROMACS tool for high-throughput MM-PBSA calculations. Journal of chemical information and modeling, 54(7), 1951-1962.

Kurczab R, Śliwa P, Rataj K, Kafel R, \& Bojarski A. J. (2018). Salt Bridge in Ligand-Protein Complexes-Systematic Theoretical and Statistical Investigations. Journal of Chemical Information and Modeling, 58(11), 2224-2238.

Lodish H, Berk A, Zipursky S. L, Matsudaira P, Baltimore D, Darnell J. (2000). Neurotransmitters, synapses, and impulse transmission. In Molecular Cell Biology. 4th edition. WH Freeman.

Morris G. M, Huey R, Lindstrom W, Sanner M. F, Belew R. K, Goodsell D. S, Olson A. J. (2009). AutoDock4 and AutoDockTools4: Automated docking with selective receptor flexibility. Journal of computational chemistry, 30(16), 2785-2791.

Orchard I. (1982). Octopamine in insects: neurotransmitter, neurohormone, and neuromodulator. Canadian Journal of Zoology, 60(4), 659-669.

Souza P. C. T, Puhl A. C, Martínez L, Aparício R, Nascimento A. S, Figueira A. C. M, Polikarpov I. (2014). Identification of a new hormone-binding site on the surface of thyroid hormone receptor. Molecular Endocrinology, 28(4), 534-545.

Srinivasan J, Cheatham T. E, Cieplak P, Kollman P. A, Case D. A. (1998). Continuum solvent studies of the stability of DNA, RNA, and phosphoramidate- DNA helices. Journal of the American Chemical Society, 120(37), 9401-9409.

Vanommeslaeghe K, Hatcher E, Acharya C, Kundu S, Zhong S, Shim J, MacKerell Jr A. D. (2010). CHARMM general force field: A force field for drug-like molecules compatible with the CHARMM all-atom additive biological force fields. Journal of computational chemistry, 31(4), 671-690.

Wang W, Kollman P. A. (2000). Free energy calculations on dimer stability of the HIV protease using molecular dynamics and a continuum solvent model. Journal of molecular biology, 303(4), 567-582.

William Tank A, Lee Wong, D. (2011). Peripheral and central effects of circulating catecholamines. Comprehensive Physiology, 5(1), 1-15.

Yu W, He X, Vanommeslaeghe K, MacKerell Jr A. D. (2012). Extension of the CHARMM general force field to sulfonyl-containing compounds and its utility in biomolecular simulations. Journal of computational chemistry, 33(31), 2451-2468. 